\title{
Parque público e criminalidade: preditores ambientais da percepção de (in)segurança
}

\section{Public park and crime: environmental predictors of the perception of (in)security}

\author{
Isângelo Senna (orcid.org/0000-0002-7002-3350) ' \\ Ana Maria Nogales Vasconcelos (orcid.org/0000-0001-7589-107X) ${ }^{2}$ \\ Fabio Iglesias (orcid.org/0000-0002-2217-5296)3
}

\begin{abstract}
Resumo
O modelo da prevenção criminal pelo design ambiental (Crime Prevention through Environmental Design - CPTED) enfatiza como elementos do ambiente podem inibir ou induzir a ocorrência de vários tipos de delitos e a percepção de insegurança. Isso é especialmente estratégico em locais de uso múltiplo e de livre acesso, como no caso dos parques públicos em grandes cidades. O objetivo desta pesquisa foi testar o papel preditivo de variáveis especificadas pela CPTED sobre a percepção de segurança, com base em um survey in loco com 126 usuários de parque público, incluindo itens sobre hábitos, ocorrências criminais, avaliação do ambiente e variáveis sociodemográficas. Modelos de regressão linear hierárquica mostraram que sexo, experiências de vitimização e a avaliação da infraestrutura funcionaram como variáveis antecedentes adequadas. Os resultados são discutidos no contexto do cenário criminal brasileiro, da inércia da população e, sobretudo, das possibilidades de intervenção em políticas públicas de segurança.
\end{abstract}

Palavras-chave: Prevenção criminal pelo design ambiental. CPTED. Segurança pública. Percepção de segurança. Parques públicos.

\footnotetext{
Abstract

1 Universidade de Brasília, Brasília, Brasil. E-mail: isangelosenna@gmail.com.

2 Universidade de Brasília, Brasília, Brasil. E-mail: nogales@unb.br.

3 Universidade de Brasília, Brasília, Brasil. E-mail: fabiglesias@gmail.com.
}

The model of Crime Prevention through Environmental Design (CPTED) emphasizes how elements of the environment may inhibit or induce the occurrence of various types of crime and the perception of insecurity. This is especially strategic in places of multiple uses and free access, as in the case of public parks in big cities. The objective of 
this research was to test the predictive role of variables specified by CPTED on the perception of security, based on a survey in loco with 126 public park users, including items on habits, criminal occurrences, environmental assessment and sociodemographic variables. Hierarchical linear regression models showed that sex, experiences of victimization, and the evaluation of infrastructure worked as appropriate background variables. The results are discussed in the context of the Brazilian criminal scenario, the inertia of the population and, above all, the possibilities of intervention in public policies for security.

Keywords: Crime prevention through environmental design. CPTED. Public security. Perception of security. Public parks.

Fatores tais como características físicas do ambiente e percepção de insegurança estão intimamente relacionados à violência urbana, a qual, por sua vez, tem figurado como um dos principais desafios para a gestão pública no Brasil, com reflexos, inclusive, para a saúde pública (Vasconcelos, Santana, Borges, Couto \& Fonsêca, 2017). Entretanto, as medidas levadas a efeito pelo Estado brasileiro vêm se mostrando paradoxais e insuficientes para a contenção da criminalidade e a redução da sensação de insegurança na população. Por um lado, a ênfase das ações estatais recai sobre o modelo tradicional de segurança pública, baseado na dicotomia polícia versus marginalidade. Nele, o policial é eficiente apenas quando responde prontamente às chamadas de emergência e efetua a prisão de delinquentes (Skolnick \& Bayley, 2002). Por outro lado, visando à redução da criminalidade, também se apregoa o investimento em polícia comunitária, na assistência a comunidades carentes e em medidas voltadas para pessoas socialmente vulneráveis. Contudo, de uma forma ou de outra, esbarra-se na máxima de que o combate ao crime é um monopólio do Estado.

As aspirações por reformas na maneira como as agências de segurança pública lidam com o crime também estão presentes na literatura internacional. Bayley (2008) destaca a instituição de sistemas de colaboração entre polícia e sociedade civil como a forma mais promissora para a ocorrência de inovações em matéria de prevenção criminal. Autoridades e comunidade acadêmica vêm apontando em direção à superação do modelo tradicional, por 
meio da identificação e da promoção de novas alternativas para a segurança pública. Atendendo a um clamor por "novas direções" e "políticas públicas alternativas" (Costa, 2004), a prevenção criminal pelo design ambiental, internacionalmente conhecida pela sigla CPTED (Crime Prevention Through Environmental Design), se revela uma alternativa consistente e viável às tradicionais propostas de prevenção do crime (Cozens \& Love, 2015).

O presente trabalho visa a contribuir com a produção de conhecimento sobre o uso da CTPED no Brasil, sobretudo no que se refere aos espaços públicos. Para tanto, investigou-se a percepção de segurança dos usuários de um parque público de grande popularidade em uma das capitais brasileiras, testando-se o poder preditivo das variáveis especificadas pela CPTED e de fatores sociodemográficos relevantes.

\section{Criminalidade, urbanização e CPTED}

Um dos fatores relacionados à criminalidade amplamente citado pela literatura é o processo de urbanização. No Brasil, tal como ocorreu no restante da América Latina, houve um acentuado crescimento de crimes violentos em paralelo com a rápida urbanização do país (Tulchin \& Ruthenburg, 2006). Por outro lado, vários estudos nos campos da criminologia ambiental (Brantingham \& Brantingham, 1981), da prevenção situacional do crime (Clarke \& Felson, 1993) e, mais especificamente da CPTED (Cozens \& Love, 2015), têm levado pesquisadores a analisar fatores socioambientais, a fim de reduzir a taxa de criminalidade e a percepção subjetiva de insegurança - medo do crime (Armitage, 2017; Brunton-Smith \& Sturgis, 2011).

Jane Jacobs (1961) foi uma das primeiras autoras a relacionar as variáveis "delito" e "espaço urbano". Ela sugeria que trazer as pessoas para caminhar na rua seria vital para a redução de índices de criminalidade, por meio daquilo que denominou "olhar sobre a rua". Para tanto, a autora apontou como estratégias principais a diversificação de atividades e a promoção 
de uma vida vibrante nas ruas, além da definição clara entre espaços públicos e privados (Schneider \& Kichen, 2007). Mais tarde, estudos de Oscar Newman (1976) sobre bairros populares e crimes em Nova Iorque estabeleceram uma conexão definitiva entre design urbano e índices de criminalidade. Newman propôs como solução o redesenho de blocos de apartamentos, de forma que áreas de uso comum pudessem estar sob a vigilância do público por todo o tempo. Em suas análises acerca da relação entre design do ambiente e crime em bairros populares, ele contribuiu sobremaneira com o delineamento do modelo teórico da CPTED, ressaltando quatro fatores cruciais: territorialidade, vigilância natural, imagem e inserção no ambiente (Fernandes, 2007). Contemporâneo de Newman, Ray Jeffery (1971) cunhou o termo CPTED, definindo a abordagem como o design apropriado e o uso efetivo do ambiente construído com vistas à redução do medo do crime e dos incidentes criminais e à melhoria na qualidade de vida.

Com um enfoque mais particular na territorialidade, a teoria do espaço defensável, proposta por Newman (1976), assumiu que as pessoas necessitam delimitar e defender seu território. Segundo o autor, um bom design urbano e arquitetônico despertaria o instinto de territorialidade nas pessoas, que passariam a defender seu espaço da ação de estranhos. Da mesma forma, projetos de bairros populares poderiam deixar claro quais espaços pertenceriam a quem. Alguns espaços poderiam ser completamente privados, outros poderiam ser compartilhados com a permissão dos proprietários, enquanto outros poderiam ser essencialmente públicos. Newman defendia que, ao desenvolver instinto territorial, as pessoas passariam a exercer uma espécie de vigilância prática em seu ambiente; premissa exportada também para os espaços públicos por meio da CPTED (Fernandes, 2007). Entretanto, estudos posteriores às pesquisas de Newman têm revelado que apenas as características físicas, per si, têm se mostrado insuficientes para se desenvolver o impulso de defesa territorial (Reynald, 2015).

Ao longo das últimas décadas, a CPTED vem sendo refinada do ponto de vista teórico e testada empiricamente (Cozens \& Love, 2015). Assim, por meio da CPTED, cada vez mais 
cidades no mundo todo têm investido estruturalmente e socialmente em ambientes que reduzem as oportunidades e a atratividade para a ocorrência de crimes; desencorajam comportamentos delituosos; estimulam a construção de suporte e vínculo entre seus habitantes e/ou usuários; e promovem a ocupação do espaço público por usuários legítimos (Crowe \& Fennelly, 2013). Ambientes menos susceptíveis à ação de criminosos e ao medo do crime, segundo os princípios da CPTED, têm quatro dimensões tradicionais: territorialidade, vigilância natural, controle de acesso e manutenção do espaço.

A territorialidade é um conceito que aborda tanto barreiras físicas quanto simbólicas que diferenciam o espaço público do privado, ao mesmo tempo em que salienta o uso legítimo desses locais. Por meio da vigilância natural, busca-se criar um ambiente em que há oportunidades de ser visto e poder observar o entorno, possibilitando o monitoramento de estranhos e habitantes por meio de janelas, entradas e câmeras de segurança, além de impedir que haja elementos arquitetônicos e naturais que bloqueiem a visualização de terceiros, como prédios muito altos, árvores sem poda e construções em locais indevidos. O controle de acesso diz respeito aos elementos que restringem ou controlem a entrada de estranhos a determinado local ou comunidade; seriam exemplo desses controles formais e informais a presença de definições espaciais (cercas e sinalização), seguranças e jardinagem. Pela manutenção do espaço, entende-se que ambientes que aparentam estar sendo cuidados têm poucas chances de serem depredados ou invadidos por assegurar que há alguém responsável por aquele local (Matsunaga, 2016).

Princípios adicionais da CPTED têm sido advogados por outros autores (Reynald, 2015), incluindo a justaposição geográfica, o fortalecimento dos alvos e o suporte ativo. O primeiro diz respeito a locais ao redor de um determinado ambiente que trazem maior segurança ou riscos de ordem criminal, um exemplo disso seriam residências próximas a determinados perfis de rodovia, que as tornariam mais suscetíveis de ser potenciais alvos de criminosos (Barclay et al., 1996). Já o fortalecimento do alvo visa a aumentar os esforços que um determinado agressor teria que empreender ao tentar cometer o ato criminoso (p. ex., portas com cadeados, 
cofres, etc.). Por sua vez, por suporte ativo, entende-se que é possível tornar um espaço público seguro pelo encorajamento de seu uso, aumentando-se o fluxo de pessoas legitimamente envolvidas nas atividades realizadas no espaço.

\section{Percepção de insegurança e uso de parques públicos}

Muito mais do que a violência real, é o medo de ser vítima de crime que causa mais impacto na forma como as pessoas pensam, sentem e se comportam (Gabriel \& Greve, 2003). Decisões importantes do quotidiano levam em conta a percepção de (in)segurança vivenciada pelos indivíduos. Isso vai da escolha do local de lazer com os filhos ao ponto comercial em que o empreendedor estabelecerá um novo negócio. Igualmente, o medo de ser vítima de criminosos constitui um dos principais fatores que levam as pessoas a evitar determinadas regiões de sua cidade e a se afastar dos espaços públicos (Armitage, 2017; Dantas, Persijn \& Silva Júnior, 2007; Guedes, Cardoso \& Agra, 2012; Hale, 1996; Kitchen \& Schneider, 2007; Loukaitou-Sideris \& Eck, 2007). Isso é especialmente notório no caso de parques públicos, considerando sua popularidade, as oportunidades de lazer gratuito que oferecem a várias classes sociais e seu papel estratégico na dinâmica do próprio funcionamento das grandes cidades (Arredondo et al., 2013; Carbone et al., 2015; Jorgensen, Ellis \& Ruddell, 2013).

Um dos fatores preponderantes para o retorno dos usuários aos parques públicos é a mitigação do ciclo que envolve desordens físicas e sociais; percepção de insegurança; e crime (Arredondo et al., 2013). A literatura revela que entre as principais variáveis que geram ou ativam a percepção de insegurança nas pessoas estão justamente as desordens físicas e sociais (Brunton-Smith \& Sturgis, 2011 ; Foster, Giles-corti \& Knuiman, 2010; Gainey, Alper \& Chappell, 2011; Gray, Jackson \& Farrall, 2011; Pitner, Yu \& Brown, 2012; Pitner, Yu \& Brown, 2013). Parques com a prevalência de práticas antissociais, malcuidados, que possuem abrigos para potenciais agressores e iluminação deficiente (Jorgensen, Ellis \& Ruddell, 2013), por exemplo, constituem locais que despertam a percepção de insegurança. Essas características afastam a 
comunidade dos parques e atraem grupos que deles fazem uso ilegítimo, como é o caso do tráfico de drogas, da prostituição, entre outros.

Ademais, a percepção de insegurança não se manifesta uniformemente entre os sexos e faixas etárias. Mulheres tendem a apresentar maiores escores de medo do crime do que homens, enquanto a percepção de insegurança aumenta com a idade (Brunton-Smith \& Sturgis, 2011 ; Foster, Giles-corti \& Knuiman, 2010; Gainey, Alper \& Chappell, 2011 ; Jorgensen, Ellis \& Ruddell, 2013; Silva \& Beato, 2013; Greve, Leipold \& Kappes, 2017). Outra variável com impacto na percepção de segurança é o histórico do indivíduo (ou de pessoas próximas) com alguma vitimização pregressa (Brunton-Smith \& Sturgis, 2011; Gainey, Alper \& Chappell, 2011; Gray, Jackson \& Farrall, 2011; Pitner, Yu \& Brown, 2013). Já a presença de outros usuários geralmente constitui fator redutor da percepção de insegurança (Jorgensen, Ellis \& Ruddell, 2013).

Em que pese a relevância do tema, são raros os trabalhos, não só no Brasil, mas em língua portuguesa, de modo geral, que se ocupam sobre a percepção de insegurança (Guedes, Cardoso \& Agra, 2012). Ainda assim, mesmo aqueles que o fazem não costumam fundamentar suas argumentações em evidências empíricas, muitas vezes resumindo a pesquisa a revisões da literatura. Uma exceção notável é a pesquisa realizada por Silva e Beato (2003), que testou dados demográficos como renda, sexo e idade em face de dados contextuais, tais como medo do crime, coesão social e desordens físicas, em bairros de Belo Horizonte. Outro raro exemplo é o estudo de Pimentel, Gunther e Black (2012), os quais encontraram correlação entre o medo do crime e a quantidade de exposição a programas de televisão no Brasil.

Considerando a investigação da percepção de segurança dos usuários de um parque público, com enfoque em variáveis construídas a partir da racional da CPTED, objetivou-se mais especificamente: a) identificar como os usuários avaliam as condições gerais de segurança no parque; b) verificar a diferença de escore entre os sexos para avaliação da percepção de segurança; c) avaliar os elementos que os usuários consideram prioritários para a segurança (características físicas do espaço; comportamento dos frequentadores; segurança privada; ou presença da Polícia Militar); d) aferir se os entrevistados percebem a relação entre design 
ambiental e segurança do local; e, por fim, e) verificar se, em face de irregularidades, os respondentes buscam soluções nas autoridades, Organizações não Governamentais ou por meio de denúncias à imprensa.

\section{Método}

\section{Participantes}

Participaram da pesquisa 126 pessoas, in loco, em um parque público urbano, sendo 82 homens (65\%), representando uma amostra não probabilística, com abordagem direta, em horários variados, incluindo horários noturnos em todos os dias da semana. A média de idade foi de 31,08 anos $(D P=12,04)$. Oitenta e quatro entrevistados $(67 \%)$ responderam ter nível superior ou, ao menos, frequentar ou ter frequentado algum curso de graduação.

\section{Locus}

Trata-se de um parque urbano planejado, em área central de uma capital brasileira, que constitui um dos fatores para sua escolha como recorte territorial. Assim como muitos outros, ele é caracterizado por uma considerável incidência de desordens e delitos, que ocupam espaço na mídia e frequentemente são associados a demandas por um incremento da presença policial. O local inclui quadras de esportes, lagos artificiais, parquinho de diversões, pistas de caminhada e ampla área de estacionamentos, com uma frequência de milhares de usuários todos os dias. 


\section{Instrumento}

Como instrumento de coleta, elaborou-se um questionário individual constituído de duas partes: a) perfil socioeconômico dos entrevistados; e b) percepção dos usuários do parque quanto à segurança geral no local, considerando as características físicas do ambiente.

As perguntas da segunda parte foram construídas a partir do conceito de CPTED. Visouse a preservar a relação entre os objetivos da pesquisa e os conceitos pesquisados (Günther \& Pinheiro, 2008). Consideraram-se para a elaboração do questionário as principais estratégias adotadas em CPTED, garantindo-se evidências de validade teórica e validade de face para os itens. Antes da composição final dos itens e de seu formato, eles foram testados em um estudo piloto com 100 respondentes, incluindo ambulantes, comerciários e comerciantes, trabalhadores do parque e usuários.

Para se aferir o quesito percepção de segurança, perguntou-se aos entrevistados: "Como você se sente em relação à segurança no parque?" Apresentou-se no formato de uma escala de cinco categorias ( 1 - "totalmente inseguro" - a 5 - "totalmente seguro"). Também se solicitou aos respondentes que ordenassem as ações, segundo o grau de importância (1 - mais relevante - a 4 - menos relevante), que poderiam contribuir para o aumento de sua segurança no parque, envolvendo intensificação da presença da PM, investimento em segurança privada (vigilantes), adoção de uma postura pessoal mais preventiva e melhoria na estrutura física (iluminação, cercas, poda de árvores e calçamento).

\section{Procedimentos}

Todas as entrevistas foram realizadas nas proximidades dos estacionamentos com maior fluxo de pessoas, ao longo do mês de fevereiro. Os questionários foram impressos de 
quatro formas distintas, que se diferenciavam pela ordenação das ações listadas na questão, para diminuir o viés da escolha dos entrevistados. O fato de não ser possível o levantamento preciso da população de frequentadores do parque e de que um esquema de seleção de amostra probabilístico oneraria a pesquisa em tempo e recursos, justificaram a escolha do plano amostral por conveniência (Barbetta, 2008). Para a análise, não foram consideradas as perguntas rasuradas, em branco ou com mais de uma alternativa assinalada, quando apenas uma era permitida.

\section{Plano de análise dos dados}

Foram utilizados procedimentos estatísticos descritivos e inferenciais, por meio de regressões lineares para testar o poder preditivo das variáveis principais, assim como testes $\mathrm{t}$ para comparar as médias nos agrupamentos dos participantes.

\section{Resultados}

Inicialmente, são sumarizados os elementos de estatística descritiva das variáveis envolvidas, configurando uma caracterização da amostra e a definição de critérios para as análises. Em seguida, são relatadas as estatísticas inferenciais que permitiram testar as principais relações entre as variáveis, para verificação das hipóteses especificadas nos objetivos.

\section{Estatísticas descritivas}

As respostas permitiram classificar, inicialmente, os participantes em frequentadores regulares (67\%) e esporádicos (33\%). Observou-se ainda que $72,5 \%$ dos frequentadores 
esporádicos do parque são frequentadores de fins de semana. Entre os entrevistados frequentadores regulares $(n=84), 51 \%$ disseram frequentar o parque antes do amanhecer ou após o anoitecer. Os entrevistados revelaram predileção aos esportes individuais (37\%), passeios com família e amigos (31\%) e prática de esportes coletivos (24\%). Quanto ao principal meio de acesso, $61 \%$ relataram usar automóvel próprio.

Sobre problemas detectados na estrutura física do parque, $44 \%$ dos entrevistados afirmaram já haver se deparado com lâmpadas queimadas ou deficientes; $38 \%$, com cercas danificadas; $37 \%$ apontaram problemas no calçamento, e $46 \%$ identificaram problemas relativos a mato alto ou árvores sem poda. Outros problemas citados pelos frequentadores foram sujeira e equipamentos quebrados (14\%). Ademais, $17 \%$ comunicaram a alguma autoridade pública; $2 \%$, a ONGs; e 5\%, à imprensa algum problema encontrado no cuidado e/ou manutenção do local. Quanto à vitimização, $17 \%$ informaram acerca de assalto a transeunte; $21 \%$, roubo/furto de/em automóvel; $14 \%$, violência física; $6 \%$, violência sexual; e $7 \%$, outros eventos, como violência verbal, assédio e homicídio. Como opções prioritárias, 42\% apontaram a presença da Polícia Militar; 25\%, melhorias na condição do espaço; e 20\%, a "adoção de postura mais preventiva por parte dos próprios entrevistados"; enquanto $12 \%$ informaram acreditar que o investimento em segurança privada pudesse ser a melhor solução para a melhoria da segurança no parque.

\section{Estatísticas inferenciais}

Homens $(M=3,40, D P=0,85)$ relataram mais sensação de segurança que mulheres $(M$ $=2,95, D P=0,87), t(122)=2,74, p=0,007, d=0,50$. Os respondentes que não relataram histórico de vitimização $(M=3,41, D P=3,43)$ também apresentaram mais percepção de segurança em relação aos que passaram por essa experiência $(M=2,95, D P=0,89), t(123)=$ $2,83, p=0,005, d=0,51$. Não se observaram diferenças significativas na percepção de segurança entre aqueles que frequentam o parque nos períodos diurno ou noturno. 
Para testar o poder preditivo das variáveis sexo, vitimização e avaliação geral da estrutura sobre a percepção de segurança, foram utilizados modelos de regressão linear. Foram verificados os principais pressupostos para esse tipo de análise, tendo-se concluído que não houve multicolinearidade das variáveis antecedentes (tolerâncias acima de 0,2 e VIFs abaixo de 10), que a variável de avaliação geral da infraestrutura revelou normalidade na sua distribuição e que os resíduos do modelo também apresentaram normalidade.

Utilizando-se o método hierárquico para entrada das variáveis, verificou-se que o modelo composto por sexo (Beta $=-0,23$ ), vitimização (Beta $=-0,22$ ) e avaliação da infraestrutura geral (Beta $=0,31$ ) explicou 19\% da variância da percepção de segurança. Assim, a categoria mulher e a categoria ter histórico de vitimização (preditores categóricos) representaram diminuição dos escores na percepção de segurança. Já a avaliação da infraestrutura (variável contínua) representou aumento dos escores nessa percepção.

Em um modelo separado, testou-se o poder preditivo de duas dimensões da CPTED, vigilância natural (representada por iluminação) e manutenção do ambiente (representada por poda e jardinagem), sobre a percepção de segurança. Nesse caso, verificou-se que somente a iluminação foi um preditor significativo (Beta $=0,43$ ), explicando $22 \%$ da variância do fenômeno. A manutenção não se manteve como preditora, depois de controlada a variância devida à vigilância natural.

\section{Discussão}

O objetivo desta pesquisa foi testar o papel preditivo de algumas variáveis especificadas pelo modelo da CPTED sobre a percepção de segurança dos usuários de um parque público. De modo sumário, verificou-se que as variáveis sexo, histórico de vitimização e avaliação geral da estrutura foram antecedentes significativos da percepção de segurança, confirmando, para o contexto brasileiro, achados da CPTED em outros países (Arredondo et al., 2013). Por sua vez, elementos relacionados à vigilância natural revelaram produzir mais efeito sobre a percepção 
de segurança que itens relacionados à manutenção do espaço. Essa é uma relação que não parece ter sido especificada anteriormente na literatura, já que as pesquisas empíricas geralmente se ocupam isoladamente sobre essas variáveis.

Os entrevistados avaliaram como positivas as condições gerais de segurança no parque, porém, conforme se verifica largamente na literatura da área (Jorgensen, Ellis \& Ruddell, 2013), percebeu-se uma maior preocupação com a insegurança por parte das mulheres. De um lado, essa discussão envolve, inevitavelmente, as representações sociais da mulher na sociedade (Vieira \& Amaral, 2013). Por outro, as diferenças de sexo são aparentes na forma como um e outro se relacionam com o ambiente, ainda que não se discutam questões objetivas de vulnerabilidade. Desde a mais tenra infância, na maioria das culturas, as meninas são encorajadas a serem mais cuidadosas e menos exploradoras que os garotos. As mulheres também tendem a ser mais atentas ao ambiente ao seu redor, principalmente no que toca ao perigo (Bechtel \& Churchman, 2003). Esses resultados sugerem que o planejamento do espaço deve levar em consideração as diferenças de sexo e buscar congruência no atendimento das duas diferentes demandas. De todo modo, permite-se inferir que os usuários, em geral, se sentem seguros no parque, com avaliações relativamente altas, a despeito dos problemas identificados.

A seu turno, quando os respondentes foram instados a classificar os elementos que consideram mais importante para a segurança do local, houve grande ênfase na presença da Polícia Militar. Tal predileção poderia estar baseada na representação social da polícia como solução prioritária para os problemas relacionados à criminalidade (Porto, 2009). De fato, comumente as políticas públicas para segurança se baseiam na premissa de que atividade criminal e desordem podem ser mais bem impedidas com a presença visível da polícia nas ruas e a prisão imediata de pessoas que infringem a lei (Skolnick \& Bayley, 2002), algo justamente questionado pela CPTED. Mas, por outra perspectiva, a segunda maior opção para o aumento da segurança no local foi a melhoria na estrutura física do Parque. Assim, conclui-se que os 
respondentes, de fato, percebem que há relação entre características físicas do ambiente e criminalidade, mesmo indiretamente.

Destacou-se nos resultados uma incongruência entre irregularidades encontradas no espaço e a inação por parte dos respondentes. Notou-se também que a frequência com que se reportam atos de violência foi duas vezes maior que a frequência de denúncias de irregularidades relativas à configuração física do local. Enquanto foram listadas mais de 200 irregularidades na estrutura física do parque, apenas $5 \%$ foram reportadas (a algum ente público, privado ou do terceiro setor) para que pudessem de alguma forma contribuir para a superação desses problemas. À luz dos princípios de CPTED, mais especificamente do conceito de reforço territorial ou territorialidade, tais dados sugerem a ausência de uma maior apropriação do parque por seus usuários. Essa é uma tendência de valor estratégico, haja vista que territórios não residenciais, como parques, tendem a ser mais suscetíveis à incidência de delitos. Ressalta-se que uma das razões para isso se assenta na facilitação das atividades delitivas, devido, justamente, à ausência de territorialidade ou de controle social (Aragonés \& Amérigo, 2010).

Se as pessoas estabelecem a conexão entre características do espaço e criminalidade e se utilizam desse espaço regularmente, por que não se sentiriam responsáveis por sua defesa, conservação e melhoria? Uma possível resposta para esse questionamento estaria no fato de as intervenções em espaços públicos muitas vezes levarem em consideração apenas o processo de planejamento, per si, o processo de tomada de decisões e os aspectos políticos correlatos. Entretanto, as necessidades das pessoas que seriam alvo do planejamento muitas vezes ficam à margem desse processo (Carbone et al., 2015; Lima \& Vasconcelos, 2006). Essa, possivelmente, seria outra razão para a baixa apropriação do espaço público por parte de seus usuários. Alternativamente, Bechtel e Churchman (2003) advogam que o planejamento dos espaços deve partir do nível micro (usuário) para então alcançar o macro (gestores públicos), em direção à maior participação de diferentes grupos de pessoas. No mesmo sentido, a comunidade poderia conhecer: a) a forma como os responsáveis pelo planejamento operam; b) o planejamento em 
nível macro; e c) as pressões sob as quais os planejadores atuam. Por conseguinte, esse esforço aproximaria cidadãos e autoridades, despertando nos primeiros um maior senso de pertencimento e participação.

Semelhante parceria decisória, alicerçada nas práticas da CPTED, encontra-se institucionalizada, entre outras localidades, em nações como Austrália e Nova Zelândia (Clancey, Lee \& Fisher, 2012). Em ambos os países, não há alteração temporária ou permanente no espaço urbano sem prévia consulta à comunidade afetada. Nesse cenário, a participação da sociedade em assuntos de interesse comum já é tomada como essencial. Outro exemplo exitoso de emprego da CPTED, com vistas ao retorno de frequentadores de parques públicos, pode ser visto em um programa realizado em San Diego - EUA (Arredondo et al., 2013). Tendo em vista que parecem ainda desconhecidos no Brasil, é fundamental o desenvolvimento de estudos empíricos, como o presente, para a investigação de fenômenos in loco e busca de evidências de validade ecológica (Bronfenbrenner, 1996).

No tocante ao conceito de vigilância natural, os entrevistados reportaram problemas quanto à iluminação, mato alto e podas de árvores no parque. Ambas as situações comprometem a possibilidade de as pessoas poderem ver e ser vistas; ou seja, violam o princípio da vigilância natural da CPTED. Chama a atenção que os respondentes frequentadores do parque no período noturno sentem-se tão seguros quanto os frequentadores do período diurno, enquanto grande parte dos entrevistados também frequenta o ambiente antes ou depois do nascer do sol, em situação de limitada luz natural. Porém, essa variável, isoladamente, não seria suficiente para determinar a qualidade da iluminação do parque. Estudos específicos, principalmente sob o enfoque de arquitetura e urbanismo, devem ser desenvolvidos nesse sentido.

A pesquisa permite supor que a ampla prática de atividades físicas no parque estudado (patinação, corrida, caminhada, etc.) contribui positivamente para a promoção da vigilância natural, pois operacionaliza a proposta de Jacobs (1961) quanto à intensificação no uso dos espaços públicos, aplicando o chamado "olhar sobre a rua". Inclusive, em estudo anterior 
envolvendo usuários de parques públicos, foi reportado menor medo do crime em face de ambientes compartilhados com outras pessoas, mesmo se tratando de desconhecidos (Jorgensen, Ellis \& Ruddell, 2013). Em sentido oposto, o acesso prioritário ao Parque por meio de transporte individual comprometeria a integração da área com o restante da cidade e mesmo entre seus múltiplos ambientes. Na CPTED, integração e conectividade dizem respeito ao movimento de pessoas entre diferentes áreas urbanas, efetuado com o maior número possível de alternativas (a pé, por bicicleta, por automóveis particulares ou transporte público). Locais altamente conectados encorajam o fluxo de pessoas e contribuem para melhores resultados de prevenção, sobretudo na perspectiva da vigilância natural. Outra consequência da opção principal dos usuários por transporte privado poderia estar refletida nos tipos de delitos reportados. Os delitos relacionados a veículos particulares ocupam o topo da lista de queixas, o que pode ser mitigado com a incrementação da vigilância natural e o rearranjo de elementos relacionados ao controle natural de acessos. O mesmo vale para os crimes de caráter patrimonial, que são crimes de oportunidade.

Em face dos resultados da pesquisa, entre as principais técnicas de CPTED propostas por Crowe e Fennelly (2013), sugere-se o desenvolvimento das seguintes ações: a) alocar eventos e reuniões em locais com vigilância natural e controle de acesso; b) desenvolver atividades com vistas à promoção de vigilância natural, incrementando a sensação de segurança para os usuários e de risco para ofensores; c) superar a distância e o isolamento por meio da melhoria das comunicações e de um ordenamento eficiente (ex:: telefones de emergência, percursos para pedestres, transporte tipo "trenzinho", etc.); e d) estabelecer barreiras naturais tendentes a se evitar o conflito entre atividades.

Com vistas a ações efetivas envolvendo a CPTED, é essencial que haja a participação da comunidade desde a fase de diagnóstico, e ao longo do processo decisório quanto às intervenções a serem realizadas, até o monitoramento dos resultados. Para que isso ocorra, comunidade, agentes públicos e policiais precisam se comunicar nos mesmos termos. A capacitação dos atores relevantes para a prevenção criminal e redução do medo do crime no 
conhecimento e operacionalização dos princípios da CPTED viria ao encontro dessa necessidade.

\section{Considerações finais}

A percepção de insegurança da população em face da criminalidade urbana constitui um dos temas mais discutidos na sociedade moderna. Entretanto, as formas usuais de enfrentamento do problema, por parte do Estado brasileiro, vêm sendo insuficientes para devolver a tranquilidade à população e conter a escalada da violência no país. A prevenção criminal pelo design do ambiente (CPTED), calcada na premissa de que o crime envolve variáveis elementares (lei, vítima, agressor e local), incorpora estratégias que extrapolam as abordagens tradicionais. O usuário é o melhor conhecedor de seu habitat e o primeiro interessado que o ambiente à sua volta lhe proporcione bem-estar. Daí tem-se o conceito de territorialidade, a qual move as pessoas a zelar, vigiar, coibir atitudes antissociais ao seu alcance ou, quando necessário, acionar o poder público para fazê-lo. Ao lado da territorialidade, outras estratégias da CPTED podem ser a vigilância natural, o controle de acesso e a manutenção do espaço.

Foram sugeridas estratégias que possibilitem aos frequentadores do parque se apropriar daquele espaço, de forma que possam participar de sua conservação, manutenção e melhoria. O envolvimento do público nas ações de planejamento de intervenções no local, somado a ações de polícia comunitária, por exemplo, poderia unir esforços nesse sentido (Skoolnick \& Bayley, 2002). Deve-se desenvolver, assim, uma ampla agenda de pesquisa no Brasil sobre a CPTED, com múltiplos métodos, que incluam a análise do comportamento de frequentadores (mapeamento comportamental), a realização de entrevistas em profundidade com usuários, administradores, prestadores de serviço e policiais que atuam em parques, mas também sobre a relação que se estabelece entre todos esses atores. 


\section{Referências}

Armitage, R. (2017). Design, Crime and the Built Environment. In N. Tilley \& A. Sidebottom (Eds.). Handbook of Crime Prevention and Community Safety (pp. 234-253). New York: Routledge.

Aragonés, J. I., \& Amérigo, M. (Coord.). (2010). Psicologia Ambiental. Madrid: Ediciones Pirâmide.

Arredondo, E., Mueller, K., Mejia, E., Rovira-Oswalder, T., Richardson, D., \& Hoos, T. (2013). Advocating for Environmental Changes to Increase Access to Parks: Engaging Promotoras and Youth Leaders. Health Promotion Practice, 14(5), 759-766.

Barbetta, P. A. (2008). Estatística aplicada às Ciências Sociais. Florianópolis: UFSC.

Barclay, P., Buckley, J., Brantingham, P. J., Brantingham, P. L., \& Whinn-Yates, T. (1996). Preventing Auto Theft in Suburban Vancouver Commuter Lots: Effects of a Bike Patrol. Crime Prevention Studies, 6, 133-61.

Bayley, D. H. (2008). Police Reform: Who Done it?. Policing \& Society, 18(1), 7-17.

Brantingham, P. J., \& Brantingham, P. L. (Ed.). (1981). Environmental Criminology. Beverly Hills: Sage Publications.

Brunton-Smith, I., \& Sturgis, P. (2011). Do Neighborhoods Generate Fear of Crime?: an Empirical Test Using the British Crime Survey. Criminology, 49(2), 331-369.

Bechtel, R. B., \& Churchman, A. (Ed.). (2003). Handbook of Environmental Psychology. New York: Wiley.

Bronfenbrenner, U. (1996). A ecologia do desenvolvimento humano: experimentos naturais e 
planejados. Porto Alegre: Artes Médicas.

Carbone, A. S., Coutinho, S., Viggiani, M., Tomerius, S., \& Philippi Junior, A. (2015). Gestão de áreas verdes no município de São Paulo: ganhos e limites. Ambiente \& Sociedade, 18(4), 201-220.

Clancey, G., Lee, M., \& Fisher, D. (2012). Crime Prevention through Environmental Design (CPTED) and the New South Wales Crime Risk Assessment Guidelines: a Critical Review. Crime Prevention and Community Safety, 14(1), 1-15.

Clarke, R. V. G., \& Felson, M. (Ed.). (1993). Routine Activity and Rational Choice. New Brunswick: Transaction Publishers.

Costa, A. T. M. (2004). Entre a lei e a ordem: violência e reforma nas polícias do Rio de Janeiro e Nova York. Rio de Janeiro: FGV.

Cozens, P., \& Love, T. (2015). A Review and Current Status of Crime Prevention through Environmental Design (CPTED). CPL Bibliography, 30(4), 393-412.

Crowe, T. D., \& Fennelly, L. J. (2013). Crime Prevention through Environmental Design. Amsterdam: Elsevier.

Dantas, G. F. L., Persijn, A., Silva Júnior, Á. P. (2007). O medo do crime. O Alferes, 22(62), 11 49.

Fernandes, L. F. (2007). Jacobs, Newman e C. Ray Jeffery: contributos para a prevenção da criminalidade. In M. Valente (Coord.). Urbanismo segurança e lei (Tomo I, pp. 33-60). Coimbra: Edições Almedina.

Foster, S., Giles-Corti, B., \& Knuiman, M. (2010). Neighbourhood Design and Fear of Crime: a Social-Ecological Examination of the Correlates of Residents' Fear in New Suburban 
Housing Developments. Health \& Place, 16(6), $1156-1165$.

Gabriel, U., \& Greve, W. (2003). The Psychology of Fear of Crime: Conceptual and Methodological Perspectives. British Journal of Criminology, 43(3), 600-614.

Gainey, R., Alper, M., \& Chappell, A. T. (2011). Fear of Crime Revisited: Examining the Direct and Indirect Effects of Disorder, Risk Perception, and Social Capital. American Journal of Criminal Justice, 36(2), 120-137.

Gray, E., Jackson, J., \& Farrall, S. (2011). Feelings and Functions in the Fear of Crime: Applying a New Approach to Victimisation Insecurity. British Journal of Criminology, 51, 75-94.

Greve, W., Leipold, B., \& Kappes, C. (2017). Fear of Crime in Old Age: a Sample Case of Resilience?. The Journals of Gerontology, 72(1), gbw169.

Guedes, I., Cardoso, C., \& Agra, C. (2012). Medo do crime: revisão conceptual e metodológica. In C. da Agra (Org.). A criminologia: um arquipélago interdisciplinar (pp. 213-248). Porto: Universidade do Porto.

Günther, H., \& Pinheiro, J. Q. (Org.). (2008). Métodos de pesquisa nos estudos pessoaambiente. São Paulo: Casa do Psicólogo.

Jacobs, J. (1961). The Death and Life of Great American Cities. New York: Random House.

Jeffery, C. R. (1972). Crime Prevention through Environmental Design. Criminology, 10, 191.

Jorgensen, L. J., Ellis, G. D., \& Ruddell, E. (2013). Fear Perceptions in Public Parks: Interactions of Environmental Concealment, the Presence of People Recreating, and Gender. Environment and Behavior, 45(7), 803-820.

Hale, C. (1996). Fear of Crime: a Review of the Literature. International Review of Victimology, 4(2), 79-150. 
Kitchen, T., \& Schneider, R. H. (2007). Crime Prevention and the Built Environment. New York: Routledge.

Lima, H. M., \& Vasconcelos, L. (2006). Integração da participação no processo de tomada de decisão referente a projectos de Engenharia. Ambiente \& Sociedade, 9(2), 71-82.

Loukaitou-Sideris, A., \& Eck, J. E. (2007). Crime Prevention and Active Living. American Journal of Health Promotion, $21(4$ suppl), 380-389.

Matsunaga, L. H., Senna, I., \& Iglesias, F. (2016). Construção de um inventário para identificação de fatores ambientais relacionados ao crime em locais públicos. In Anais do I Congresso Internacional do Nordeste Psicologia Jurídica e Direito Penal, Salvador, BA.

Newman, O. (1976). Defensible Space: Crime Prevention through Urban Design. New York: Coolier Books.

Pimentel, C. E., Gunther, H., \& Black, P. U. V. (2012). Acessando o medo do crime: um survey por meio da internet. Psicologia Argumento, 30(69), 411-421.

Pitner, R. O., Yu, M., \& Brown, E. (2012). Making Neighborhoods Safer: Examining Predictors of Residents' Concerns about Neighborhood Safety. Journal of Environmental Psychology, $32(1), 43-49$.

Pitner, R. O., Yu, M., \& Brown, E. (2013). Which Factor Has More Impact?: an Examination of the Effects of Income Level, Perceived Neighborhood Disorder, and Crime on Community Care and Vigilance among Low-Income African-American Residents. Race and Social Problems, 5(1), 57-64.

Porto, M. S. G. (2009). Brasília, uma cidade como as outras?: representações sociais e práticas de violência. Sociedade e Estado, 24(3), 797-826. 
Reynald, D. M. (2015). Environmental Design and Crime Events. Journal of Contemporary Criminal Justice, $37(1), 71-89$.

Skolnick, J., \& Bayley, D. (2002). Policiamento comunitário: questões e práticas do mundo. São Paulo: Edusp.

Tulchin, J. S., \& Ruthenburg, M. (Ed.). (2006). Toward a Society under Law: Citizens and their Police in Latin America. Washington DC: Woodrow Wilson Center Press.

Vasconcelos, D. C. D., Santana, I. O. D., Borges, L. C., Couto, R. N., \& Fonsêca, P. N. D. (2017). Adaptação e evidências de validade e precisão do questionário de atitude frente à violência na escola. Gerais: Revista Interinstitucional de Psicologia, 1O(1), 13-24.

Recebido em: 17/5/2018

Aprovado em: 19/9/2018 